\title{
Periodic Orbit Theory for Rydberg Atoms in External Fields
}

\author{
P. A. Dando, T. S. Monteiro, and S. M. Owen \\ Department of Physics and Astronomy, University College London, Gower Street, London WC1E 6BT, United Kingdom
}

(Received 21 July 1997)

\begin{abstract}
Although hydrogen in external fields is a paradigm for the application of periodic orbits and the Gutzwiller trace formula to a real system, the trace formula has never been applied successfully to other Rydberg atoms. We show that spectral fluctuations of general Rydberg atoms are given with remarkable precision by the addition of diffractive terms. Previously unknown features in atomic spectra are exposed: there are new modulations that are neither periodic orbits nor combinations of periodic orbits; "core shadowing" generally decreases primitive periodic orbit amplitudes but can also lead to increases. [S0031-9007(98)05735-4]

PACS numbers: $32.60 .+\mathrm{i}, 03.65 . \mathrm{Sq}, 05.45 .+\mathrm{b}$
\end{abstract}

Periodic orbit theory, in the form of the Gutzwiller trace formula (GTF) [1], provides the most powerful framework for the semiclassical quantization of chaotic systems. It is more than a decade since it was first shown that the GTF provides a quantitative description of the oscillations in the density of states of highly excited hydrogen atoms in magnetic fields [2]. However, the trace formula has never been applied successfully to any other species of singly excited (Rydberg) atoms. This is clear from comparisons between accurate quantal spectra since the spectral amplitudes for nonhydrogenic atoms differ substantially from those of hydrogen [3].

Much effort has been expended in developing closed orbit theory [4-7], the semiclassical theory which describes photoabsorption by atoms in external static fields. By matching semiclassical waves to Coulomb waves near the nucleus, the photoabsorption strength is obtained as a sum of contributions from only those orbits that close at the nucleus. In contrast, all periodic orbits contribute to the GTF (the eigenvalue spectrum). It was shown that, provided core-scattered waves are included consistently in the matching procedure, closed orbit theory can be applied to general atoms in fields [8-10]. But, for nonhydrogenic atoms one finds additional modulations of $O(\sqrt{\hbar})$ or higher relative to the hydrogenic contributions - the "combination recurrences"- that are due to sums of closed orbits. Contributions from the harmonics of closed orbits are reduced in amplitude through core shadowing [8-10] but those associated with the first traversals of the primitive orbits are unaffected.

However, closed orbit theory does not account, even qualitatively, for the observed differences between atomic species for the eigenvalue spectrum, i.e., the density of states, $\rho(E)=-\operatorname{Im} \operatorname{Tr} G(E) / \pi$. In the density of states - quantitatively well-described by periodic orbit theory for hydrogen-amplitudes of primitive periodic orbits can vary substantially between atoms, in contrast with closed orbit theory. Also, the modulations associated with combinations of periodic orbits appear at different orders in $\hbar$ relative to closed orbit theory.
Rydberg atoms and molecules in the field-free case are described by quantum defect theory (QDT), one of the most widely used theories in atomic physics. In QDT, the effects of a multielectron core are described by a set of phase shifts, or "quantum defects," $\delta_{l}$, in each partial wave, $l$. In the limit when the quantum defects vary smoothly with $l$, they can be related to the classical precession angle of the Kepler ellipse by $\Theta=2 \pi d \delta_{l} / d l$. For many atoms only the lowest partial waves have nonzero quantum defects. For example, for even parity lithium, $\delta_{0} \simeq 0.4 \pi$ and $\delta_{l \geq 2} \simeq 0$ while for helium $\delta_{0} \simeq 0.3 \pi$ and $\delta_{l \geq 2} \simeq 0$. In this case, variation of $\delta_{l}$ with $l$ is clearly not smooth.

In this Letter, we present an approach that, for the first time, combines the Gutzwiller trace formula with quantum defect theory and, hence, sheds new insight on the classical interpretation of quantum defects. Our approach yields simple analytical expressions for the differences in amplitudes for general nonhydrogenic atoms. We compare the new semiclassical results with full quantal calculations and, for well isolated orbits, find them to be extremely accurate, for example, to within about $1 \%$ at $\hbar \simeq 0.01$.

A surprising finding, predicted by theory and confirmed by quantal results, is that, although the amplitudes of the primitive orbits are mostly reduced, as one would expect from the idea of core shadowing, in the nonhydrogenic case they can also increase. This is shown below to be due to a dephasing between diffractive (core) and geometric (Coulomb) contributions. Combinations of periodic orbits appear with order at least $\hbar$. Most significantly, new modulations appear that are not combinations of real periodic orbits but are rather pure diffractive orbits. They pass through the core and are made periodic by the diffraction. We emphasize that all these effects are accurately described by the diffractive periodic orbit theory.

The periodic orbit theory of diffraction was developed recently for Hamiltonians with discontinuities [11-13]. For our purposes, a good example of a diffractive system is the cardioid billiard, which has a single sharp vertex. In 
this case, periodic orbits are decomposed into two kinds: those that do not intersect the vertex (geometric orbits) and those that do (diffractive orbits). The density of states has been shown to be obtained as a sum:

$$
\rho(E)=-\frac{1}{\pi} \operatorname{Im} \operatorname{Tr} G_{g}(E)-\frac{1}{\pi} \operatorname{Im} \operatorname{Tr} G_{D}(E) .
$$

The first (geometric) term yields the ordinary GTF. The trace, over the second (diffractive) contribution has been shown to be $[11,13]$

$$
\operatorname{Tr} G_{D}(E)=\sum_{p} \frac{T_{p}}{i \hbar} \prod_{n} d(n) G\left(q_{n}, q_{n+1} ; E\right),
$$

where $T_{p}$ is the total sum of periods taken over the paths between the vertices and $d(n)$ is the diffraction constant which depends on the type of diffraction. Equation (2) encapsulates the important result that the trace integral taken between the $n$th and $(n+1)$ th vertices is proportional to the Green's function between those points.

We apply diffractive periodic orbit theory to our atomic systems by treating the nonhydrogenic core as a diffractive source. The crucial step is to obtain an expression for the diffractive constant $d$ in terms of quantum defects. To this end, we consider an incoming Coulomb wave, $\psi_{\text {Coul }}^{(-)}$, which approaches the atomic core from infinity at an angle, $\theta_{f}$, to the $z$ axis. On reaching the core, $\psi_{\text {Coul }}^{(-)}$produces a scattered wave, $\psi_{\text {scatt }}$, which feeds outgoing semiclassical waves along periodic orbits; $\psi_{\text {scatt }}$ can be decomposed into an outgoing Coulomb wave together with a core-scattered wave [5]: $\psi_{\text {scatt }}(r, \theta)=$ $\psi_{\text {Coul }}^{(+)}(r, \theta)+\psi_{\text {core }}^{\theta_{f}}(r, \theta)$. The Coulomb scattered wave is strongly back focused along $\theta \simeq \theta_{f}$ and can be written in closed form [5]. Our first approximation consists of equating $\psi_{\text {Coul }}^{(+)}$with the source for geometric paths (i.e., the usual GTF). The core-scattered wave $\psi_{\text {core }}$, arising from the incoming wave at angle $\theta_{f}$, is equated with the source of diffractive semiclassical waves. At a radius, $r_{0} \simeq 50 \mathrm{bohr}$, we express $\psi_{\text {core }}^{\theta_{f}}$ in a partial-wave expansion which, for $m=0$ is [5]

$$
\begin{aligned}
\psi_{\text {core }}^{\theta_{f}}= & \left(\frac{2 \pi^{2}}{r^{3}}\right)^{1 / 4} \\
& \times \sum_{l=0}^{\infty} Y_{l 0}^{*}\left(\theta_{f}, 0\right) Y_{l 0}(\theta, 0) e^{-i 3 \pi / 4}\left(e^{2 i \delta_{l}}-1\right),
\end{aligned}
$$

where $\delta_{l}$ are the quantum defects. Finally, we take $d$ to be the fractional amplitude scattered by the core:

$$
d\left(\theta_{i}, \theta_{f}\right)=\psi_{\text {core }}^{\theta_{f}}\left(r_{0}, \theta_{i}\right) / \psi_{\text {Coul }}^{(-)}\left(r_{0}, \theta_{f}\right) .
$$

All calculations and comparisons with fully quantal spectra presented here have been carried out for $s$-wave scattering (appropriate for atoms such as lithium or helium which are used frequently in experiments of atoms in fields). So, below $\delta \equiv \delta_{0}$ and, in this case, $\psi_{\text {core }}^{\theta_{f}}$ is isotropic. However, generalization to odd parity or atoms with multiple quantum defects is straightforward.
We consider the specific example of Rydberg atoms in a static magnetic field of strength, $\gamma$ (atomic units). The quantum spectra are calculated at a constant scaled energy $\epsilon=E \gamma^{-2 / 3}$, that is for fixed classical dynamics. Quantum mechanically, we calculate a set of eigenvalues, $\gamma_{i}^{-2 / 3}$, corresponding to different effective $\hbar$ [14]. Below, $\hbar$ denotes $\gamma^{1 / 3}$.

For Rydberg atoms in a magnetic field, the best studied periodic orbits are the straight line orbit perpendicular to the field, $R_{1}$, and the "balloon" orbit, $V_{1}^{1}$. The wellknown Garton-Tomkins orbit [15], $R_{1}$, is responsible for the quasi-Landau oscillations observed in $m=1$ atomic spectra near the ionization limit at energy spacing $\sim 1.5 \hbar \omega$; these were the first observed "footprints" of periodic orbits in a real physical system. The balloon orbit dominates $m=0$, odd- $l$ spectra with oscillations of spacing $\sim 0.64 \hbar \omega$. The effect of the core on the orbit parallel to the field, $V_{1}$, is relatively weak $[4,8,9]$. The periodic orbit labeling terminology of Ref. [16] is used throughout.

For the case of $s$-wave scattering, each diffractive contribution in Eq. (2) is

$$
\begin{aligned}
d G= & \sqrt{\hbar}\left(e^{2 i \delta}-1\right)\left|\frac{2 \pi}{m_{12}} \sin \frac{\theta_{i}}{2} \sin \frac{\theta_{f}}{2}\right|^{1 / 2} \\
& \times e^{i(S / \hbar-\mu \pi / 2-\pi / 4)},
\end{aligned}
$$

and, in effect, represents the contribution of a pure diffractive orbit. Note the additional phase of $-\pi / 4$ relative to an equivalent geometric primitive periodic orbit.

Now we see that the amplitude of each nonhydrogenic primitive periodic orbit actually arises from the interference between two contributions with the same action but a different phase: a geometric one of the Gutzwiller form weighted by the trace of the stability matrix, $M$, in the usual manner, i.e., for the $p$ th orbit $A_{H}^{p}=\left(\pi S_{p}\right) / \sqrt{\left|2-\operatorname{Tr} M_{p}\right|}$, and a diffractive one, given by Eq. (5), following the same path and of similar action but weighted by $1 / \sqrt{m_{12}}$, where $m_{12}$ is an element of $M$. This contrasts with the cardioid billiard where a typical contribution is either pure geometric or pure diffractive.

We can easily show that the fractional reduction of amplitude for a primitive periodic orbit of a nonhydrogenic atom relative to that of hydrogen is

$$
\begin{aligned}
& A_{\delta} / A_{H} \\
& \quad=\sqrt{1+4 \mathcal{R}^{2} \sin ^{2}(\delta) \hbar-4 \mathcal{R} \sin (\delta) \sin (\delta+\phi) \sqrt{\hbar}},
\end{aligned}
$$

where, in general,

$$
\mathcal{R}=A_{H}^{-1} \sqrt{\frac{32 \pi}{m_{12}} \sin \left(\theta_{i} / 2\right) \sin \left(\theta_{f} / 2\right)} .
$$

All parameters, e.g., initial and final angles, $\theta_{i}, \theta_{f}$, refer to the particular primitive orbit under consideration. The 
fractional reduction or increase is of $O(\sqrt{\hbar})$. In general, $\phi=-\pi / 4$. However, $R_{1}$ runs along a boundary of the fundamental symmetry domain and so requires special treatment: when stable, with winding number $\nu$, we find $\phi=-\pi / 4-\nu \pi / 2$ and $\mathcal{R}=2 \sqrt{\left(\pi / m_{12}\right)} \sin (\pi \nu)$. The most important correction in Eq. (6) is the $\sqrt{\hbar}$ term. This is zero for $\delta=-\phi$ and positive for $\sin (\delta+\phi)<$ 0 , leading to an increased amplitude for a primitive periodic orbit. In contrast, in closed orbit theory, the main photoabsorption source term and the core-scattered terms do not have this $\pi / 4$ dephasing.

Harmonics of primitive orbits also have further contributions from product terms of $O(\hbar)$. However, product terms also give additional weak contributions at actions that are sums of periodic orbits. For example, a two-orbit combination has amplitude

$$
\begin{aligned}
8 \pi^{2}\left(S_{1}+S_{2}\right) & \frac{\left(e^{2 i \delta}-1\right)^{2}}{\left|m_{12}^{1} m_{12}^{2}\right|^{1 / 2}} \\
& \times\left|\sin \frac{\theta_{i}^{1}}{2} \sin \frac{\theta_{f}^{1}}{2} \sin \frac{\theta_{i}^{2}}{2} \sin \frac{\theta_{f}^{2}}{2}\right|^{1 / 2} .
\end{aligned}
$$

In Fig. 1(a) we show Fourier transforms of the oscillatory part of the even- $l, m=0$, eigenvalue spectra for hydrogen and lithium $(\delta=0.4045 \pi)$ in a static magnetic field at constant scaled energy $\epsilon=-0.2$, and with $n=\gamma^{-1 / 3}=\hbar^{-1}$ ranging from 60 to 120. In Fig. 1(b) we plot the "difference" spectrum obtained by coherently subtracting the Fourier transform of the hydrogenic spectrum from that of lithium; this exposes the diffractive contributions and eliminates contributions from periodic orbits which do not pass through the core. For comparison, we also plot a semiclassical difference spectrum obtained by summing all terms of order $\sqrt{\hbar}$ and $\hbar$; agreement is excellent. The discrepancy in $D_{2}$ is due to the effects of bifurcations that are not taken into account in the semiclassical calculation presented here.

We can see that for lithium the amplitudes of $R_{1}$ and its harmonic $R_{2}$, as well as $V_{1}^{1}$ and other orbits are substantially reduced. There are additional small peaks which correspond accurately to sums of periodic orbits. Importantly, there are strong peaks (marked $D_{1}$ and $D_{2}$ ) which do not match any combination of orbits. At these scaled actions $(S \simeq 2.87$ and $S \simeq 2.94$ ) we find orbits that are closed but not periodic. For hydrogen, only orbits that are periodic in the fundamental symmetry domain contribute. Here we see that pure diffractive orbits, such as $D_{2}$, can contribute to the nonhydrogenic spectrum at $O(\sqrt{\hbar})$, so are substantially stronger than combinations of orbits. The peak at $S \simeq 2.87$ is due to an isolated closed orbit and is obtained almost exactly from Eq. (5) as seen in Fig. 1(b) [note that in Fig. 1(a) the peak associated with this orbit is masked by the peak of a periodic orbit which does not approach the nucleus]. The peak at $S \simeq 2.94$ consists of contributions from a pair of nonisolated orbits close to a bifurcation

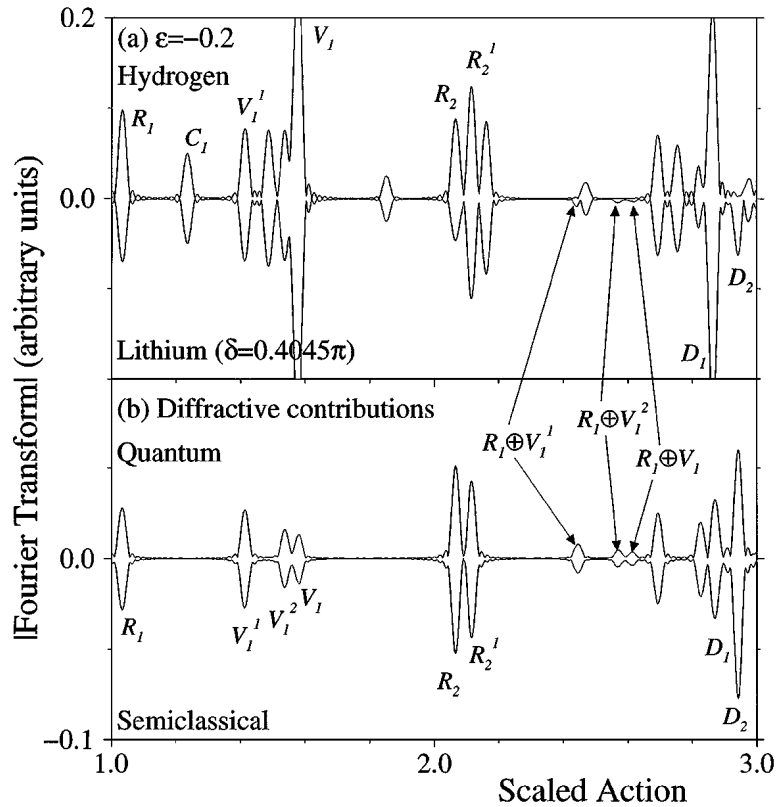

FIG. 1. (a) Comparison of Fourier transforms of the density of states for hydrogen and lithium $(\delta=0.4045 \pi)$ in a static magnetic field at constant scaled energy $\varepsilon=-0.2$ from a fully quantal calculation with average $\hbar=1 / 90$. Note the changes in amplitudes of periodic orbits and new modulations due to diffractive orbits in the lithium case. (b) Comparison between quantal and semiclassical difference spectra obtained by coherently subtracting the Fourier transforms shown in (a). This exposes the diffractive contributions to the spectrum and eliminates contributions from orbits which do not pass through the core. Shown are changes in periodic orbit amplitudes due to diffraction, diffractive combinations of two periodic orbits, and pure diffractive orbits marked $D_{1}$ and $D_{2}$. Away from bifurcations, which affect $V_{1}$ and $D_{2}$, the agreement between quantum and semiclassical calculations is excellent.

so their contribution is overestimated semiclassically. On examination of the diffractive orbits we find that they correspond to the first closure of asymmetric periodic orbits, some of which correspond to the $X_{n}$ series of "exotic orbits" [16]. In hydrogenic eigenvalue spectra such orbits contribute only at their full period, whereas in the diffractive case they appear at closure.

We have carried out a detailed study of these effects for several scaled energies to study the $\hbar$ and $\delta$ dependence of the diffractive effects. In Figs. 2(a)-2(d) we compare the fractional change relative to hydrogen between the fully quantal and semiclassical expressions for $R_{1}$ and $V_{1}^{1}$. The agreement is very good. For the $\hbar$ dependence there are fewer points for $V_{1}^{1}$ since a wide spectral range is required to resolve it from a nearby orbit. An especially interesting feature is the dephasing of $R_{1}$ relative to $V_{1}^{1}$ seen in Figs. 2(c) and 2(d). The diffractive contribution to $V_{1}^{1}$ is $-\pi / 4$ out of phase with the geometric term. As a result the amplitude exceeds that of hydrogen for $\delta \lesssim \pi / 4$ and is minimal at $\delta \simeq 0.65 \pi$. In contrast, the geometric and diffractive contributions for $R_{1}$ are almost in phase at $\epsilon=-0.275$ and remain so for a wide 

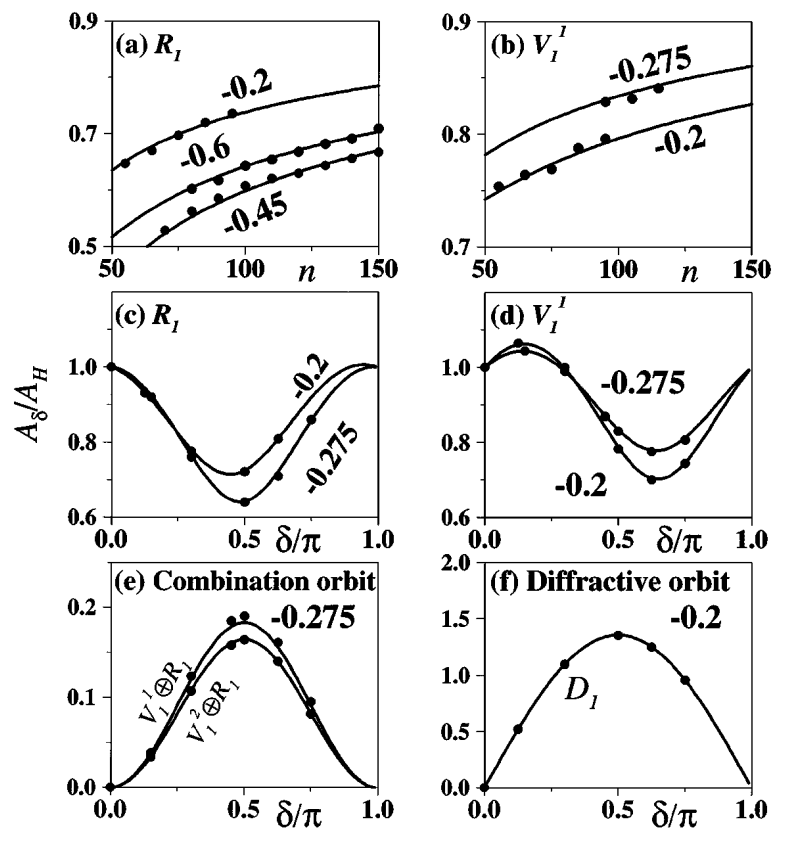

FIG. 2. Dependence of diffractive contributions on $\hbar$ and $\delta$ : comparison between quantum results (full circles) and semiclassical formula, Eq. (6), (solid line) showing near exact agreement. The vertical axis represents the ratio of nonhydrogenic to hydrogenic amplitudes. (a) Dependence of $A_{\delta} / A_{H}$ on $\hbar$ for $R_{1}$ at $\epsilon=-0.2,-0.6$, and -0.45 . (b) Same as (a) for $V_{1}^{1}$ at $\epsilon=-0.2$ and -0.275 . (c) Dependence of $A_{\delta} / A_{H}$ on quantum defect, $\delta$, for $R_{1}$. (d) Same as (c) for $V_{1}^{1}$ : note the dephasing relative to $R_{1}$ and that the amplitude exceeds the hydrogenic value for $\delta<\pi / 4$. (e) and (f) dependence of contributions of combination and diffractive orbits on $\delta$. Here the ratio relative to the first contribution of the circular orbit, $C$, is shown. As predicted by the theory, combination orbits show a $\sin ^{2} \delta$ behavior while diffractive orbits follow a $\sin \delta$ curve.

range of scaled energies about $\epsilon \simeq-0.3$, where the orbit undergoes its $2: 1$ resonance with $\nu \simeq 0.5$.

In Figs. 2(e) and 2(f) we investigate the combination orbits and the diffractive orbit that appears at $\epsilon=-0.2$ for $S \simeq 2.87$. In this case we plot the ratio of amplitudes relative to the first peak of $C$, the circular orbit, a periodic orbit that does not pass through the nucleus and, hence, is unaffected by the diffraction. In both cases the agreement is very good.

In conclusion, we have shown that periodic orbit theory (the GTF) may be applied to all singly excited atoms as successfully as for hydrogen by bringing in the effects of QDT in the form of diffractive corrections. Also, to our knowledge, this is the first demonstration of a diffractive effect in a real system, since previously diffraction has been applied only to model problems such as billiards. Although we show explicit results for $s$-wave scattering in lithium and helium, our method is applicable generally to other Rydberg atoms and molecules in external fields.

An interesting recent calculation [17] treated the photoabsorption of general atoms within the framework of the standard theory using a model potential. Then, the observed closed orbit modulations were modeled by superposing thousands of very unstable orbits. Hence, the issue of whether the dynamics of nonhydrogenic atoms at moderate scaled energies is an instance of chaos (i.e., very unstable motion) or an effect "beyond periodic orbits," such as diffraction, remains open. Our work addressed this issue.

Currently, there is added interest in diffractive systems since they have very recently been associated with a new class of intermediate level statistics ("half-Poisson") [18]. Recently, eigenvalue statistics for rubidium in fields were investigated experimentally [19] and shown to be nearer the Gaussian orthogonal ensemble (GOE) limit than comparable hydrogenic results. Hence, given that spectroscopic resolution exceeds mean level spacing, experimental verification of diffractive effects in the eigenvalue spectrum, for example, the presence of the " $D$ " modulations, is, in principle, possible.

We are indebted to E. B. Bogomolny, D. Delande, and J. B. Delos for helpful advice and discussions. The authors acknowledge funding from the EPSRC.

[1] M.C. Gutzwiller, Chaos in Classical and Quantum Mechanics (Springer-Verlag, New York, 1990).

[2] D. Wintgen, Phys. Rev. Lett. 58, 1589 (1987).

[3] W. Jans, T.S. Monteiro, W. Schweizer, and P. A. Dando, J. Phys. A 26, 3187 (1993).

[4] M. L. Du and J. B. Delos, Phys. Rev. A 38, 1896 (1988); 38, 1913 (1988).

[5] J. Gao, J. B. Delos, and M. Baruch, Phys. Rev. A 46, 1449 (1992); J. Gao and J. B. Delos, ibid. 46, 1455 (1992).

[6] E. B. Bogomolny, Zh. Éksp. Teor. Fiz. 96, 487 (1989) [Sov. Phys. JETP 69, 275 (1989)].

[7] G. Alber, Z. Phys. D 14, 307 (1989).

[8] P. A. Dando, T.S. Monteiro, D. Delande, and K. T. Taylor, Phys. Rev. Lett. 74, 1099 (1995).

[9] P. A. Dando, T. S. Monteiro, D. Delande, and K. T. Taylor, Phys. Rev. A 54, 127 (1996).

[10] J. A. Shaw and J. B. Delos (to be published).

[11] G. Vattay, A. Wirzba, and P.E. Rosenqvist, Phys. Rev. Lett. 73, 2304 (1994).

[12] H. Primack, H. Schanz, U. Smilansky, and I. Ussishkin, Phys. Rev. Lett. 76, 1615 (1996).

[13] H. Bruus and N. D. Whelan, Nonlinearity 9, 1023 (1996).

[14] D. Delande et al., J. Phys. B 27, 2771 (1994).

[15] W. R. S. Garton and F.S. Tomkins, Astrophys. J. 158, 839 (1969).

[16] A. Holle, J. Main, G. Wiebusch, H. Rottke, and K.H. Welge, Phys. Rev. Lett. 61, 161 (1988).

[17] B. Hüpper, J. Main, and G. Wunner, Phys. Rev. Lett. 74, 2650 (1995); B. Hüpper, J. Main, and G. Wunner, Phys. Rev. A 53, 744 (1996).

[18] E. B. Bogomolny (private communication).

[19] H. Held, Ph.D. thesis, Münich, 1997. 